\title{
At-Focus Observations of High Quality Electron Vortex Beams Created from Ferromagnetic Rods
}

\author{
Arthur M. Blackburn ${ }^{1}$, James C. Loudon ${ }^{2}$, Rodney Herring ${ }^{3}$, Ales Hrabec ${ }^{4}$, David Hoyle ${ }^{5}$ \\ ${ }^{1}$ Hitachi Cambridge Laboratory, Hitachi Europe Ltd., Cambridge, U. K. \\ ${ }^{2}$ Department of Materials Science and Metallurgy, University of Cambridge, Cambridge, U. K. \\ ${ }^{3}$ Department of Mechanical Engineering, University of Victoria, Victoria, BC, Canada. \\ ${ }^{4}$ School of Physics and Astronomy, University of Leeds, Leeds, U.K. \\ ${ }^{5}$ Hitachi High-Technologies Canada, Toronto, Canada.
}

Electron vortex beams carry orbital angular momentum and have use in electron microscopy for characterizing the magnetic and chiral properties of materials [1,2]. For these applications it is preferable to have a single isolated vortex beam to simplify the interpretation of scanning probe measurements. However, the first-used fork-like grating masks for producing vortex beams [1] produce multiple beams off-axis and thus require an additional selection aperture, ideally accompanied with additional lenses or a monochromator, to produce a single beam for scanning [3]. To avoid this requirement for additional hardware and to produce brighter vortex beams, thus increasing the collected dichroic signal strength, alternative approaches to producing vortex beams on-axis are being investigated, including: continuous thin-film phase plates; spiral-like apertures; carefully tuned electron microscope aberrations in combination with an annular aperture; and using a narrow ferromagnetic rod, magnetized along its long axis, partially inserted into the beam path $[4,5]$.

The ferromagnetic rod based approach (Fig. 1(a)) offers the advantage of producing a beam-energy independent, single electron vortex beam on-axis with high intensity. However, a potential perceived disadvantage that is experimentally addressed here is the quality of the vortex beam at focus which has not been clear in earlier observations [4], where the apparent quality of the produced beam may have been limited by the imperfect spiral-like nature of the produced phase-shift or other artefacts such as charging and dispersion in structures surrounding the rod. Other evidence of vortex beams produced by ferromagnetic rods has used defocussed probe observations, with this condition alleviating the obscuring of the beam point spread function by the finite coherence of the illumination, or equivalently by convolution with the electron source image, while also allowing beam rotation observation [5].

In this work, cantilevered ferromagnetic rods were formed by sputter deposition of $10 \mathrm{~nm} \mathrm{Co}_{50} \mathrm{Fe}_{50}$ with Ta adhesion and capping layers, upon one side of a series of micro-machined cantilevers which were subsequently coated in Au (Fig. 1(b)). Following magnetization in an external magnetic field, electron holograms were collected in the region of the rod-tip to determine the form of the electron phase-shift produced by the stray magnetic field and the flux-contained in the rod. From this we found that though not all the rods had the desired single domain arrangement and related helical phase shift, which could likely be remedied by improved fabrication processes, the majority of rods behaved in the desired manner and proved able to produce vortex electron beams.

With the sample rods in a standard transmission electron microscope sample holder, a $10 \mu$ m diameter selected area aperture was centered upon a single rod-end, employing conventional low magnification imaging (i.e. with the objective lens off) and images were collected of the focused beam, occurring about the imaging-lens diffraction plane. Images of the beam at its minimum diameter 'waist', for rods 
determined from electron holography to contain a magnetic flux $n h / e$ with $n$ near 2, 3 and 4 (Figs. 2(ac)), show the vortex nature of the beam, with a clear central minimum through focus.

The average radial intensity profile of these beams (Fig. 2(d)) shows fair agreement with a simple aberration-free model, once finite electron source size effects are included. Adding further aberrations would enable a better model fit. The source size, or the related finite coherence of the electron illumination over the region of the rod, manifest in these observations, need not dominate in higher resolution probes when greater source demagnification can readily be employed with high-brightness electron sources. Indeed, the observations presented here, which directly show that it is possible to achieve high quality vortex beams at focus with a ferromagnetic rod, indicate that it is worthwhile to investigate such arrangements to further the application of electron vortex beams for characterizing and understanding magnetic and chiral material behavior.

[1] J Verbeeck, H Tian, and P Schattschneider, Nature, 467 (2010), p. 301.

[2] A Asenjo-Garcia and F J G de Abajo, Physical Review Letters, 113 (2014), p. 066102.

[3] O L Krivanek, J Rusz, J C Idrobo, et al., Microscopy and Microanalysis, 20 (2014), p 832.

[4] A M Blackburn, J C Loudon, Ultramicroscopy, 136 (2014), p. 127.

[5] A Béché, R Van Boxem, G Van Tendeloo, et al., Nature Physics, 10 (2014) p. 26.

(a)

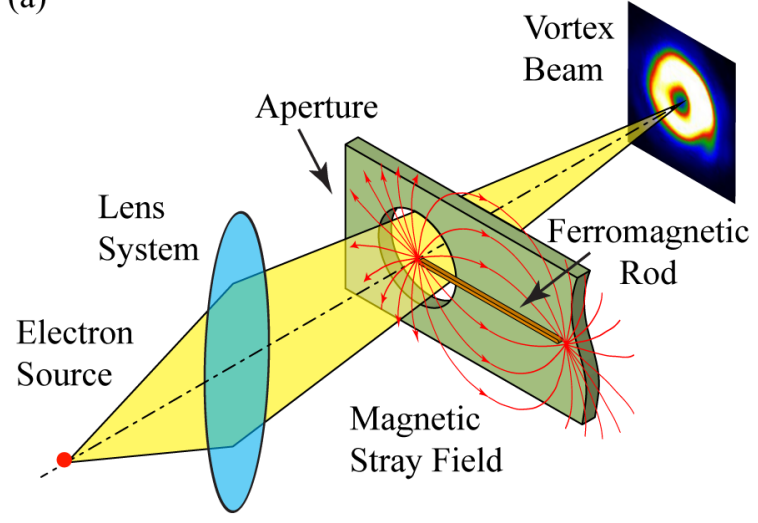

(b)

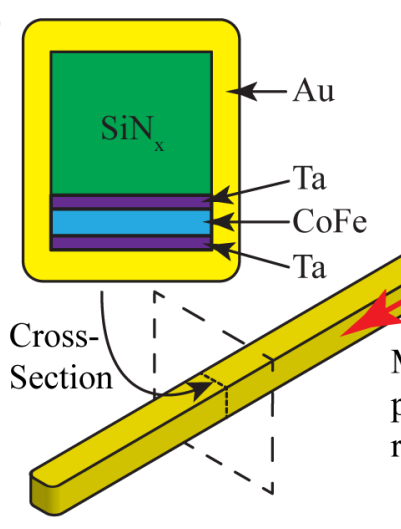

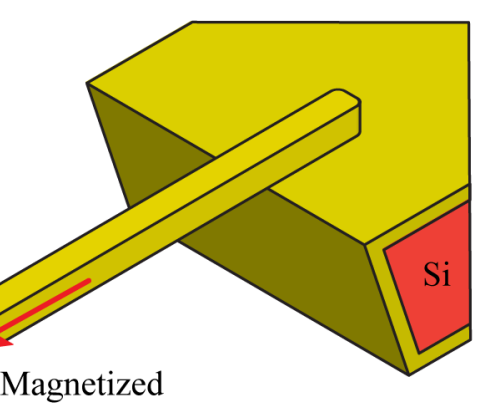

parallel to

rod long-axis

Figure 1. Schematic arrangement of (a) the ferromagnetic rod and aperture used to create an electron vortex beam, and (b) the rod and its cross-sectional composition.

(a)

$n=2$

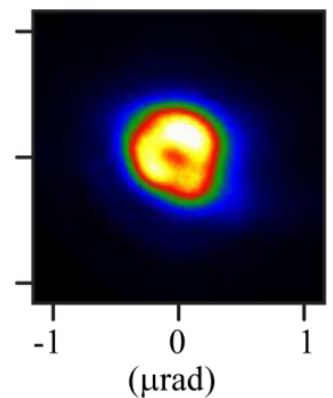

(b) $n=3$

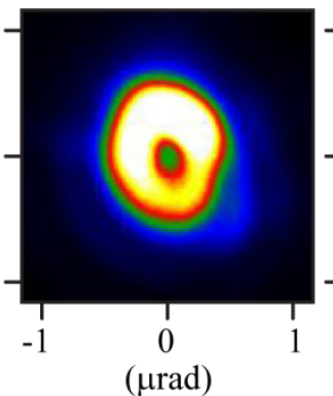

(c) $n=4$

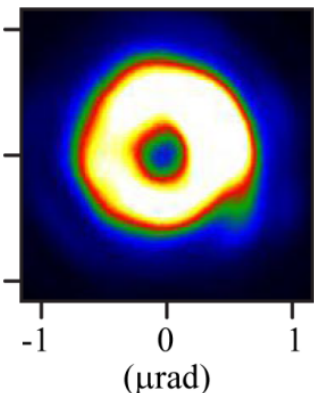

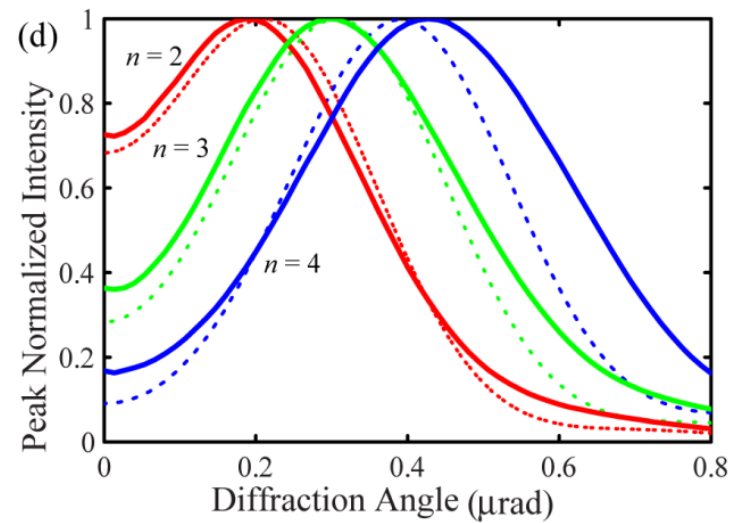

Figure 2. At focus images of $300 \mathrm{keV}$ vortex beams produced from ferromagnetic rods containing a magnetic flux of $\sim n h / e$ with $n=2,3$, and 4 (a, b, and c) and the (d) average radial intensity profiles (solid-lines) and simple aberration free modelled intensities (dashed-lines) with source convolution. 